2020-03-30

\title{
Automatic Flood Detection in Sentinel-2 Images Using Deep Convolutional Neural Networks
}

\author{
Pallavi Jain \\ pallavi.jain@tudublin.ie \\ Bianca Schoen-Phelan \\ Technological University Dublin, bianca.schoenphelan@tudublin.ie \\ Robert J. Ross \\ Technological University Dublin, robert.ross@tudublin.ie
}

Follow this and additional works at: https://arrow.tudublin.ie/scschcomcon

Part of the Artificial Intelligence and Robotics Commons

\section{Recommended Citation 3341105.3374023 \\ This Conference Paper is brought to you for free and open access by the School of Computer Sciences at ARROW@TU Dublin. It has been accepted for inclusion in Conference papers by an authorized administrator of ARROW@TU Dublin. For more information, please contact arrow.admin@tudublin.ie, aisling.coyne@tudublin.ie,gerard.connolly@tudublin.ie.}

Jain, P., Schoen-Phelan, B. and Ross, R. (2020). Automatic flood detection in Sentinel-2 images using deep convolutional neural networks. Proceedings of the 35th Annual ACM Symposium on Applied Computing (SAC '20). Association for Computing Machinery, New York, NY, USA,p.617-623. doi:10.1145/

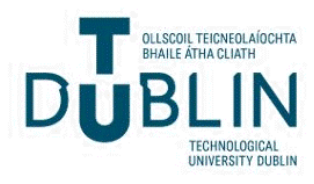




\title{
Automatic Flood Detection in Sentinel-2 Images Using Deep Convolutional Neural Networks
}

\author{
Pallavi Jain, Bianca Schoen-Phelan, Robert Ross \\ Technological University Dublin \\ Dublin, Ireland \\ \{pallavi.jain,bianca.schoenphelan,robert.ross\}@tudublin.ie
}

\begin{abstract}
The early and accurate detection of floods from satellite imagery can aid rescue planning and assessment of geophysical damage. Automatic identification of water from satellite images has historically relied on hand-crafted functions, but these often do not provide the accuracy and robustness needed for accurate and early flood detection. To try to overcome these limitations we investigate a tiered methodology combining water index like features with a deep convolutional neural network based solution to flood identification against the MediaEval 2019 flood dataset. Our method builds on existing deep neural network methods, and in particular the VGG16 network. Specifically, we explored different water indexing techniques and proposed a water index function with the use of Green/SWIR and Blue/NIR bands with VGG16. Our experiment shows that our approach outperformed all other water index technique when combined with VGG16 network in order to detect flood in images.
\end{abstract}

\section{KEYWORDS}

Remote Sensing, Flood Detection, Neural Networks, Sentinel-2

\section{ACM Reference Format:}

Pallavi Jain, Bianca Schoen-Phelan, Robert Ross . 2020. Automatic Flood Detection in Sentinel-2 Images Using Deep Convolutional Neural Networks. In The 35th ACM/SIGAPP Symposium on Applied Computing (SAC '20), March 30-April 3, 2020, Brno, Czech Republic , 7 pages. https://doi.org/10.1145/ 3341105.3374023

\section{INTRODUCTION}

Floods are common natural disasters that occur throughout the year in many locations around the globe. Flooding can occur for a myriad of reasons, for example unusually heavy rainfall, melting snow, or tsunamis, and are often exacerbated by a lack of vegetation. In most cases floods tend to be highly destructive to property and human life [11], [19] and as a result the early and accurate detection of the geographic areas affected by flood is seen as crucial. Within this context remote sensing has become very popular [26] [8], typically due to being able to show the location and severity of flooding at the same time.

Permission to make digital or hard copies of all or part of this work for personal or classroom use is granted without fee provided that copies are not made or distributed for profit or commercial advantage and that copies bear this notice and the full citation on the first page. Copyrights for components of this work owned by others than the author(s) must be honored. Abstracting with credit is permitted. To copy otherwise, or republish, to post on servers or to redistribute to lists, requires prior specific permission and/or a fee. Request permissions from permissions@acm.org.

SAC '20, March 30-April 3, 2020, Brno, Czech Republic

Q 2020 Copyright held by the owner/author(s). Publication rights licensed to ACM. ACM ISBN 978-1-4503-6866-7/20/03...\$15.00

https://doi.org/10.1145/3341105.3374023
A flood mapping is a technique to analyse the extent of flood water, and identifies its spatial distribution over the land area; these are now common place. In recent years there has been an increase in techniques proposed that aim to map water bodies [18], [29], [5], [17], [20] in order to assist with flood mapping and response. While automatic detection of floods from raw satellite image data seems like an obvious method for accurate detection, the problem is in practice non-trivial. Floods are shallow waters that are difficult to map in urban areas due to those areas being highly built-up; plus floods can be obscured by cloud coverage.

Despite the challenges in automated identification and severity mapping, we argue that satellite data nonetheless presents the best opportunity for accurate flood mapping. The Copernicus Sentinel-2 satellite project for example provides high resolution multi-spectral images with 13 bands $^{1}$ for analysis. Each band has its own characteristics, which differentiate the different geological components such as water, vegetation, clouds and land cover in terms of their reflectance values. Using this type of data we have already seen several projects that map water bodies or flood situations with varying levels of success [22], [25], [3].

Most current and historical approaches to mapping water bodies from remote sensor data has relied on the idea of water indices. Water indices are essentially hand-crafted mathematical functions which provide a measure usually based on the ratio of light reflectance at different wavelengths. These indices are helpful in highlighting areas of water relative to other objects such as vegetation or built-up area. These water indexing techniques have shown great results in order to map flood or water bodies regions. However, floods are not typical water bodies and most techniques fails to detect shallow water [20], [27]. Significant work is required in order to improve the accuracy of detection methods to minimise mis-classification, and also to increase our ability to detect the severity of flooding.

Though over the time, research has shown that appropriate combination of spectral indices are able to overcome the built-up areas and cloud shadow issues. For example Xu [29] used SWIR and Green band to overcome the built-up area, while Mishra \& Prasad [20] used the combination of Normalised difference water index (NDWI) [18] with Blue/NIR spectral indices to detect shallow water. Similarly AWEI technique by Fyesia et al. [5] helped in overcome the cloud shadow problem by calculating index using coefficient values with different bands. Such techniques are valuable as they significantly reduce the likelihood of mis-classification of an area.

Beyond the realm of flood analysis, there has been significant improvements in the accuracy of automatic visual assessment methods thanks to the growth of Deep Learning [7], [15] and its many

\footnotetext{
$\overline{{ }^{1} \mathrm{https} / / / \text { sentinel.esa.int/web/sentinel/user-guides/sentinel-2-msi/resolutions/spatial }}$
} 
architectural variations such as Convolutional Neural Networks (CNNs) [14]. One of the key differentiating factors in the success of these methods is that rather than relying exclusively on handcrafted features - comparable to water indices - they automatically learn what combinations of raw data provide the best basis for making estimates of a given target variable.

Given the relative success of CNNs across a range of domains where hand crafted features have been replaced or augmented with significant improvement of results, we argue that a systematic investigation of the application of CNNs will likely lead to significant increases in predictive power than can be gained with water index type features alone. This paper thus proposed a tiered approach where a novel water index map tuned to the particulars of the flood water challenge is fed in as base data to a deep CNN VGG16 network [24].

We proceed in Section 1.1 with a review of the most common methods that have historically been applied to water body detection, before, in Section 1.2, moving on to discuss the basic features of a CNN based approach to sensor data analysis. Following that, in Section 2 we set out our concrete study design in terms of the data used, methods investigated, and methods of evaluation. Section 3 presents the raw results along with a discussion. Finally, in Section 4 we set out our conclusions and suggestions for future work.

\subsection{Water Detection Techniques}

Traditional water body detection techniques make use of differences in electromagnetic signatures of different bodies, i.e., the relative absorption (or reflectance) of electromagnetic radiation across a range of wavelengths. The wavelengths considered typically range from the ultraviolet, down through the visible spectrum, on to near infrared (NIR) as well as short wave infrared (SWIR). The NIR band is highly absorbed by water and reflected by vegetation. This makes water appear dark in NIR images and consequently highlights it more profoundly. Similarly, the SWIR band has a lower reflectance value for water compare to vegetation or build areas.

Given the relative reflectances of different substances at different wavebands, we can design specific functions, or indices, that calculate a metric to estimate the potential for water being present or not. These water indices, similar in nature to the also common vegetation indices, provide an approximate likelihood estimation for water presence. Rather than there being one universal water index there are in practice a wide number of such indices. One well known such index is the NDWI from McFeeters [18].

$$
N D W I=\frac{\text { Green }-N I R}{\text { Green }+ \text { NIR }}
$$

As can be seen this is a relatively simple index that simply makes use of the NIR and green bands.

A similar modified NDWI (MNDWI) was later proposed by Hanqiu Xu [29], who argued that SWIR would provide a more robust method for providing segmentation in built-up areas.

$$
M N D W I=\frac{\text { Green }-S W I R}{\text { Green }+ \text { SWIR }}
$$

Both NDWI and Modified NDWI have been widely used to map water or flood areas [25], [22], [3]. Lately, various methods were proposed by either combining these water indexes, or proposing a different combination of indexing techniques in order to map water. Mapping flood in urban areas can be challenging as NDWI cannot capture shallow water areas. Additionally, shadows from varying sources are a problem [29] [5]. Several modifications of water indices have been proposed.

For example Feyisa et al. [5] proposed the automated water extraction index (AWEI). This index attempts to overcome the difficulty in identifying water in urban areas, and to solve misclassification due to shadows of mountains or buildings. This more complex functions leverages blue, green, NIR, SWIR1 and SWIR2 bands and proposed two variants: (i) shadow and (ii) non shadow. The significance here is that in flood detection urban shadows and mountain shadows can be significant, hence it could be more appropriate to use shadow version.

$$
\begin{aligned}
& A W E I_{S}=\text { Blue }+2.5 \times \text { Green }- \\
& \quad 1.5 \times(\text { NIR }+ \text { SWIR } 1)-0.25(\text { SWIR } 2)
\end{aligned}
$$

More recently, Mishra \& Prasad [20] proposed another modified NDWI index (I) to overcome the NDWI lack of separating built-up areas and the challenge of detecting shallow water. For that they added another dimensionless index using Blue and the NIR band in order to extract more detail.

$$
I=N D W I+\frac{B l u e-N I R}{B l u e+N I R}
$$

Another approach in order to reduce mis-classification due to built-up areas creates a three-dimensional feature space by utilising NDWI, Normalised difference vegetation index (NDVI), and Red Edge NDWI (RE-NDWI) [12] to detect water using supervised learning [17].

$$
\begin{gathered}
N D V I=\frac{\text { Red }-N I R}{\text { Red }+N I R} \\
R E-N D W I=\frac{\text { Green }-R E}{\text { Green }+R E}
\end{gathered}
$$

The idea here is that each of the three indices NDWI, NDVI, and Red Edge NDWI, act as high-level features that can be fed into a machine learning algorithm that can learn the higher-order functions based on combinations of these features.

While a machine learning driven approach to build higher order functions based on the low-level indices does provide a more robust approach to providing accurate flood estimates, the approach is however still dependent on the low-level indices themselves being in some way useful. However the very fact that there is such a large number of different water indices indicates that the true challenges is that simple hand crafted index functions cannot capture the variation in features that underpin an accurate identification of water bodies. Additionally, it is important to consider that most indexing techniques have been developed in order to detect water bodies in general. Therefore, flood detection might behave in a manner not anticipated, and that methods for flood detection should ideally account for specifics of flood rather than simply water detection

Rather than relying on manually crafting water indices, we argue that a automated approach to index construction is much more likely to give rise to useful estimates. In the next section we show how Deep learning and Convolutional Neural Networks in particular have great potential to provide such a mechanism. 


\subsection{Deep Convolutional Neural Network}

Deep Learning is a modern variation on the classical artificial neural network. In its basic form Deep Learning can be used for many classification and regression tasks such as flood detection by automatically learning very complex functions from raw input data to target variables. In the past ten years, deep learning methods have shown excellent performance in tasks ranging from speech recognition through to image detection and object segmentation. The key idea with supervised Deep Neural Networks is that a complex and costly initial training period can automatically be used to find optimal parameters for very complex deep functions so long as suitable training data is available. While the initial training process is expensive, the resulting trained networks are relatively cheap from a computational perspective and can easily be applied in real-time to new data.

While the basic design of a deep learning network is based only on layers of simple linear functions, there are a number of important architectural variations that are used to group together layers, share weights, and improve on performance. One important such architectural variant for our purposes is the Convolutional Neural Network (CNN), which is a multi tier network including convolutional layer, pooling layer and fully-connected layer as shown in Figure 2. The CNN takes advantage of the idea that interesting low-level features are often spatially invariant. To put this in simple terms, a line is a useful feature in object identification and lines are regardless of whether they are at the bottom left of an image or the top right. Importantly, while CNNs have historically been developed and used for traditional image data, i.e., either grey-scale or Red-Green-Blue images, they can in principle be scaled to take advantage of remote sensed data with many more spectral bands.

While the $\mathrm{CNN}$ combined with a related model component called the pooling layer, provide a basic building block for supervised training on image like data, a number of specific and detailed architectures have been proposed to take these ideas and build very deep networks that provide high-performance. These include networks such as AlexNet [13], CaffeNet [10], and VGGNet [24]. These networks are very deep and make use of a number of optimisations to provide high-performance. However, one notable challenge with these networks to train from scratch is that they require significant amounts of data to train - usually far more than is available in a specific domain such as flood detection. For this reason a number of networks have been pre-trained on alternative datasets are available for bootstrapping model development. A prominent example of such alternative dataset for pre-trained networks is ImageNet [2].

It has been shown that such pre-trained models can generally be then used to train domain specific models with small datasets [23]. Specifically for remote sensing classification tasks, it has been shown that deep CNNs can provide great performance [21], [4], [9]. Among these models VGGNet has shown outstanding results in several domain classification tasks [28], and even in remote sensing images classification [6], [9], [16].

\section{STUDY DESIGN}

Motivated by the good insights provided by water index based methods and the excellent performance of CNN based architectures for a range of imaging tasks in recent years, we propose a modelling approach to synergise the best of these two elements. In this section we outline the specifics of the approach that we take. We look first at the details of the dataset that we are using for this study, and then go on to explore some of the important aspects of the model and present the specifics of the training and evaluation methodology.

\subsection{Data}

For this study, we make use of the data provided by the MediaEval 2019 competition. Here one of the competition tasks, MultimediaSatellite, was to identify flood in time series data from Sentinal-2 images [1]. The data consists of 335 sets with 267 as development sets and 68 as test sets. Each set consists of varying 1 to 24 day time series images of before and after flood events; this provides a total 2770 images. The images are of 512 X 512 pixels in size and offer 12 bands of sentinel- 2 data. The data comes in two different sets of resolutions. The bands with $20 \mathrm{~m}$ resolution are up-scaled to $10 \mathrm{~m}$ resolution images. For this work we leveraged only the development set, as test set labelled data is not available. We thus applied our own splits to the development data as described later. Labels for the development set are binary - indicating the presence or otherwise of a flood in the given image.

Since the data provided does include information across a range of wavebands, we can immediately apply a number of water indices directly to the data to attempt to identify flooded regions. Resultant images for each of NDWI, MNDWI and AWEI, as well as the index proposed by Mishra \& Prasad [20] and Li et al.'s [17] three-dimensional feature space, can be seen in Figure 1. As noted earlier, cloud coverage and cloud shadow are two of the major problems when dealing with remote sensing images. Specifically, they are sometimes misinterpreted as actual water, while other times clouds and shallow water can have the same index value. As seen in Figures 1b, 1c, 1e, shallow water (Flood Water) is similar to partial clouds. In Figure1d only some clouds are showing, and some clouds mix with water values. Though in Figure 1f clearly differentiates between shallow water and cloud.

\subsection{Our Proposed Approach}

Our proposed model below can be described as a linear hybridisation of the classical index based approach with the CNNs. The approach attempts to combine a sensible index that is specialised for the specifics of flood identification with the generalisable features provided by a pre-trained $\mathrm{CNN}$.

To provide a sensible baseline index, let us consider the specific performance of a number of indices when benchmarked against the sample image shown in figure 1 . Here we show the histograms in figure 3 for index values calculated across all pixels in the image for Figure $4 \mathrm{a}$. The histogram in figure $4 \mathrm{c}$ shows less noise and smooth as compare to histograms for other indices in figure 3 , due to the fact that the index count reduces when water image is cloudy while pikes increases when it is cloud free as in figure 4d. Mostly cloudy part remains at \pm zero value, but stays separated from water pixels, which gives good separation to water values from all other objects.

Given the relatively good performance of threshold based models such as [20], we apply this model as a baseline for our proposed index. However, considering the cloud coverage problem in remote 




(a) Original Image

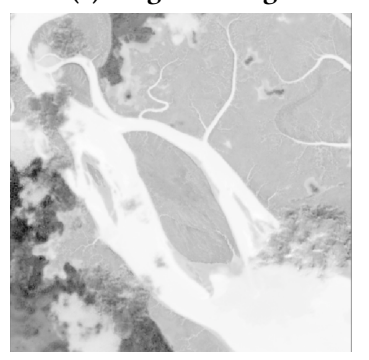

(d) AWEI [5]

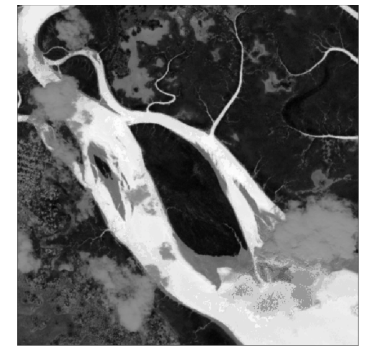

(b) NDWI [18]

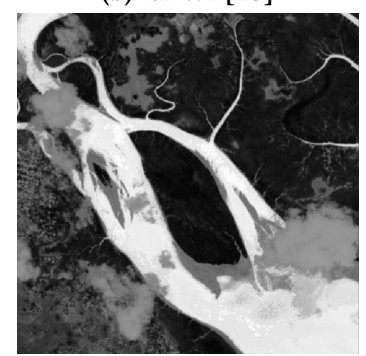

(e) Mishra et al. [20]

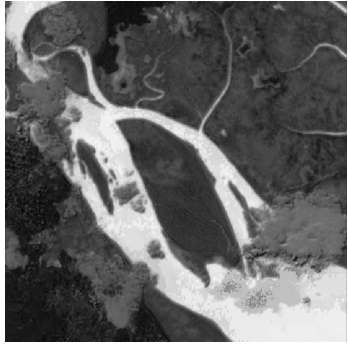

(c) MNDWI [29]

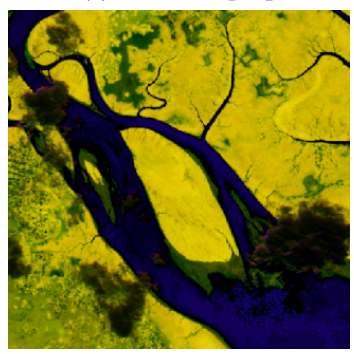

(f) Li et al. [17]

Figure 1: Different Water Index Techniques

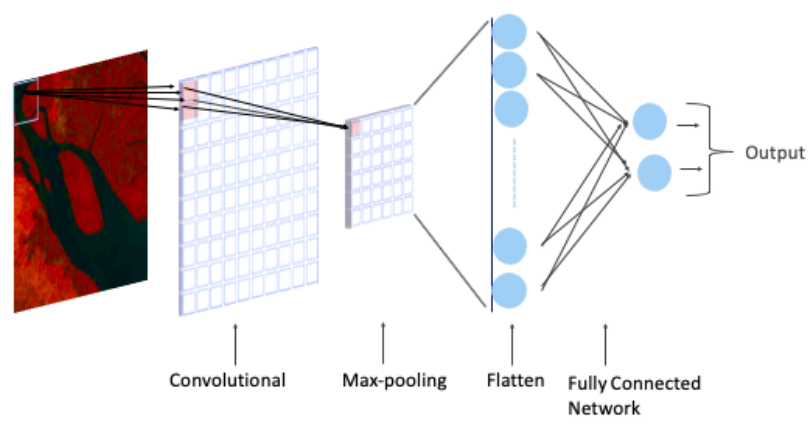

Figure 2: Convolutional Neural Network

sensing satellite images, it is good to look for its modification in indexing technique. As SWIR and Blue band are both helpful for images that present clouds, snow and penetration of thin clouds. Therefore, in our approach we replaced NDWI [18] with MNDWI [29]. Also it has been seen that blue and green bands provide the largest difference between the reflectance of water and shadow [5], which resolve the shadow issues. The resultant proposed index (PI) is as follows:

$$
P I=\frac{\text { Green }-S W I R}{\text { Green }+S W I R}+\frac{\text { Blue }- \text { NIR }}{\text { Blue }+ \text { NIR }}
$$

By using SWIR instead of NIR, the amount of noise in the image is reduced [20], apart from that due to cloud coverage the overall index values count reduces. This can be seen in figure $4 \mathrm{c}$, our approach has the lowest count index that remains positive. This is because it keeps only water pixels positive and all other as negative. In contrast all the indices in figure 3 , except Li et al. [17], show water pixels above 0 value which can be variable due to atmospheric or brightness effects. Moreover, while Li et al. [17] had a threshold that lies near 0.4 , we didn't change the axis to retain their original range in order to differentiate between water and non-water pixels. For the proposed index we also see that, for cloud free images the pixels increased in positive side as water is clearly visible. We argue that this shows our method is capable of reducing the overall impact of clouds in the image.

While our hybrid model does make use of an altered index function to provide raw data to the flood classifier, the resultant index image is also processed via a convolutional network. In particular we have made use of the VGGNet Networks that have shown great performance in ILSVRC-2014 task [24]. The VGGNet networks are one of the most popular deep CNNs for image classification and object detection. Two versions of the VGGNet are widely used: (i) VGG16 which contains 13 convolutional layers and 3 fully-connected layers, and (ii) VGG19 which consist of 16 convolutional layers and 3 fully-connected layers. This network uses $3 \times 3$ convolutional layers stacked to others to increase depth. Max pooling is used to handle the reduction of volume size. Two fully-connected layers, each with 4096 nodes, are then followed by a softmax classifier.

As noted earlier the amount of data available for tasks such as satellite based classification of flooding is limited relative to the amount of data required to train models such as VGG16 from scratch. Therefore we make use of a pre-trained network that has previously been trained on large image datasets such as ImageNet [2]. This is a form of Transfer Learning where the knowledge originally acquired for training VGGNet for the ImageNet corpus is re-used in building a classifier for a domain specific task such as our own. In such an approach the initial layers of the network usually learn very general features, while task-specific features are learned only in later layers of the network. Thus, for transferring a network to a 




(a) NDWI[18]

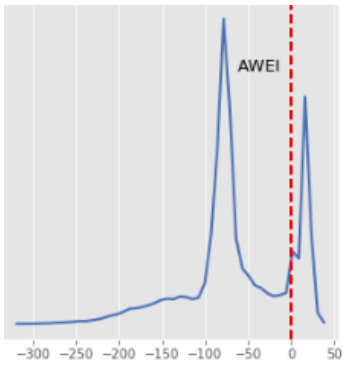

(c) AWEI [5]

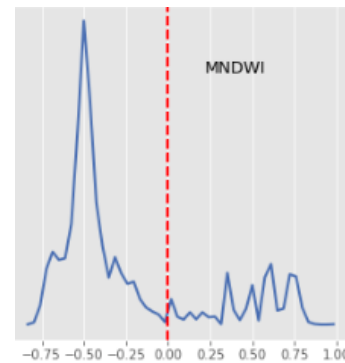

(b) MNDWI [29]

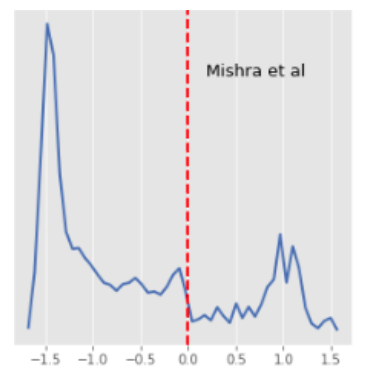

(d) Mishra et al. [20]

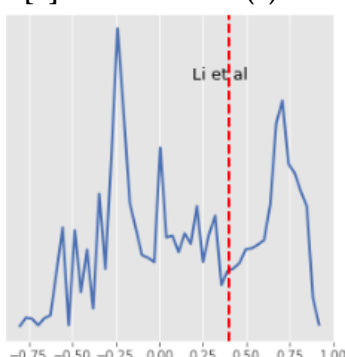

(e) Li et al. [17]

Figure 3: Each histogram shows the count of indices in corresponding image in figure 1 , and dotted vertical line shows approximate threshold to separate water and non-water indices. Figure 3a, 3b, 3c, and 3d, water indices are above zero while for figure $3 \mathrm{e}$ its above 0.4 .

specific task, we freeze the initial layers of the network and retain the later layers for our own work.

\subsection{Experimental Setup}

In our experiment setup, data pre-processing consisted of taking each dataset and for each collection of spectral images in that dataset we generate a series of water index maps. Each of these water index maps consist of a 512 x 512 array that contain the water index as calculated for each of the 6 index types we are investigating, namely, NDWI, MNDWI and AWEI, as well as the index proposed by Mishra \& Prasad [20], and Li et al.'s [17] threedimensional feature space, and our own proposed index. We split 2,208 images of the development dataset into Validation (10\%), Devtest $(10 \%)$, and $80 \%$ of data as training data. In order to increase our

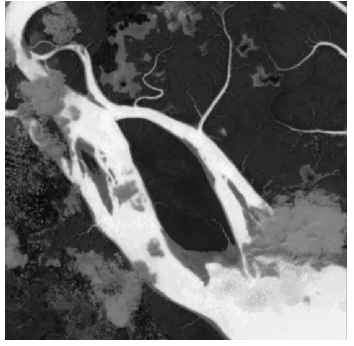

(a) Clouded Image



(c) Clouded Histogram

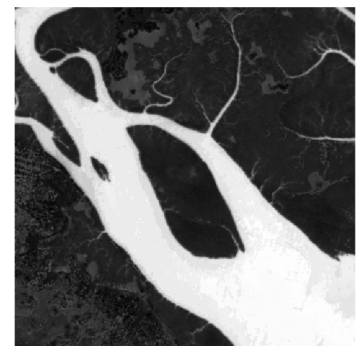

(b) Cloud Free Image

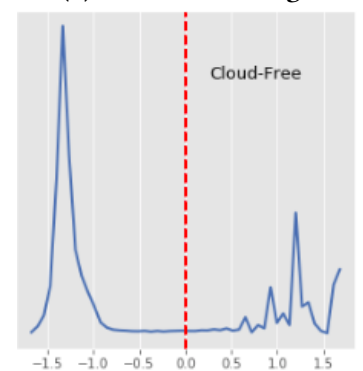

(d) Cloud Free Histogram
Figure 4: Proposed Approach

training dataset we also performed image augmentation by shifting, rotating, and flipping the image with batch size of 32 .

VGGNets like other pre-trained image processing neural networks are typically trained to work on 3 channel image data, i.e., Red Green and Blue. However in our case with water index maps are individually essentially single channel images - similar to a greyscale image but representing individual water indices. The one exception to this is Li et al.'s [17] as they used 3 dimensional feature space of water and vegetation indices such as NDWI, NDVI, and RENDWI. To work around this issue we split our single channels three ways assuming identical values for each input channel. While this is not an ideal situation given low-level features have been trained assuming colour based differences across channels, we argue that it nevertheless provides excellent performance in our tiered model.

For our work, we used model architecture with VGG16 network and fully connected layer as shown in figure 5. During training we froze the initial layers until Block 4 in the VGG16 model. Block 5 was left trainable to allow task specific features to be learned. Global average pooling was used to reduce the overfitting of the model by reducing the total parameters. After the VGG16 blocks, we used a fully connected layers of size 128 units followed by dropout layer with a dropout parameter of 0.5 , and finally a softmax layer. Rectified linear unit (ReLU) has been used as activation function and we applied the Adam optimiser to guide the training process. As our problem was a binary classification problem we used the binary cross entropy loss function to calculate the loss. For training of the model we used a learning rate of $5 \mathrm{e}-6$ and trained the model for $55 \pm 5$ epochs depending on each index best performance 


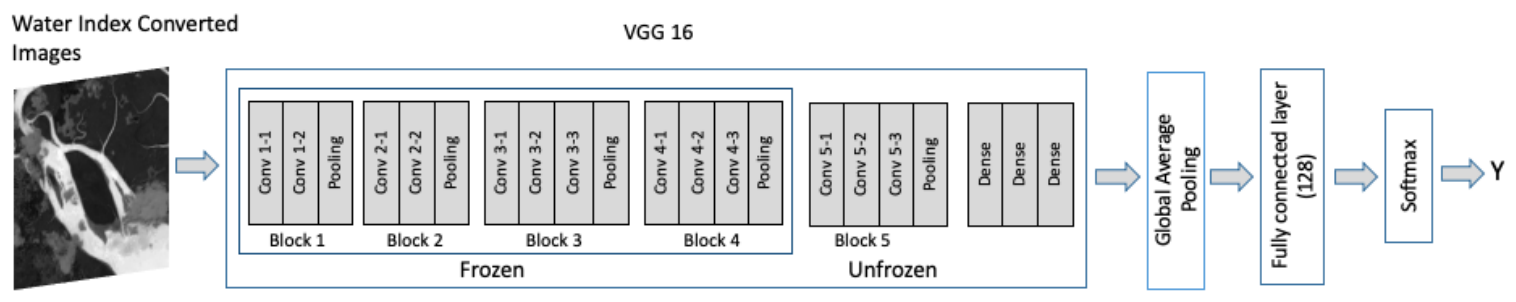

Figure 5: Network Architecture

Table 1: Evaluation of all the water indexes

\begin{tabular}{|l|l|l|l|l|}
\hline Index Type & TP & TN & F1 & Kappa \\
\hline NDWI [18] & 0.84 & 0.78 & 0.80 & 0.59 \\
MNDWI [29] & 0.76 & 0.96 & 0.88 & 0.74 \\
AWEI [5] & 0.74 & 0.90 & 0.83 & 0.66 \\
Mishra et al.[20] & 0.83 & 0.94 & 0.90 & 0.79 \\
Li et al.[17] & 0.92 & 0.84 & 0.87 & 0.71 \\
Proposed & $\mathbf{0 . 9 3}$ & $\mathbf{0 . 9 8}$ & $\mathbf{0 . 9 6}$ & $\mathbf{0 . 9 2}$ \\
\hline
\end{tabular}

\section{RESULTS}

The flood identification problem is a binary classification task. Therefore for evaluation we make use of the F1 score and Kappa. F1 score is a harmonic mean of the precision and recall performance metrics. As a harmonic mean it is thus a conservative estimate and only provides a good mean estimate if both precision and recall values are performing well. Cohen's Kappa meanwhile compares observed accuracy with expected accuracy. Kappa provides the fair comparison when the classes are imbalance or multi-class. As the data had imbalance class, it is good to look further with Kappa apart from $\mathrm{F} 1$ for the fairness of the results.

$$
F 1=2 \times \frac{\text { Precision } \times \text { Recall }}{\text { Precision }+ \text { Recall }}
$$

where Precision $=T P /(T P+F P)$ and Recall $=T P /(T P+F N)$. TP, FP, and FN are true positive, false positive, and false negative respectively.

$$
\text { Kappa }=\frac{p_{o}-p_{e}}{1-p_{e}}
$$

where $p_{0}$ is observed accuracy and $p_{e}$ is expected accuracy i.e. hypothetical expected probability of agreement.

Results from the table 1 shows that our proposed index outperforms the other index techniques when trained on VGG16 network with freezing all layers except the last one. As our approach outperformed all other index performance and index by Mishra \& Prasad [20], performed the second best. It can be said that combination of indexes can be the good idea in order to enhance the performance. For that matter Green/SWIR and Blue/NIR performed best in our case with VGG16 network architecture.

\section{DISCUSSION}

As flood mapping and monitoring is a vital aspect to organize any rescue program, automatic flood detection also carries significant importance, as it reduces manual efforts. The relatively good performance of several water index techniques, and the very strong performance of CNN based architectures such as VGGNet motivated us to systematically investigate the combination of these methods for the task of automatic flood detection. In this paper we explore different aspects of water indexing techniques and propose a modification in combining an index technique originally proposed by Mishra \& Prasad [20]. We evaluate our suggested index compared to several previously proposed approaches using supervised learning and found that our approach outperformed other indexing techniques.

Our work shows that the idea of detecting floods in real-time can be achievable when an appropriate water indexing technique is combined with the high performance deep CNN models. Mishra \& Prasad [20] originated the idea of combining two indexes to leverage benefit from both of them. As the flood water is different from normal water body, it is difficult to map shallow water. But due to recent advancement in the deep CNN models and their outstanding performance in several practical application, we can automate the flood detection process.

As deep CNNs, extract features on the go, it is important to provide them right water index. Most of the water indices showed great result in mapping but not all necessarily work well with CNNs. Considering that we replaced NDWI in Mishra \& Prasad [20] with Modified NDWI by Xu [29]. Our approach outperformed all other indices, which shows that Green/SWIR and Blue/NIR works well with deep learning and automatic detection in sentinel-2 satellite images. With a manual inspection, we also saw that our approach reduces the noise in the images and clearly distinguish between clouded and cloud free water images. In particular our index makes the water area positive but count of indexes reduces when the water image is clouded and increases when it is cloud-free. This reduces the likelihood of mis-classification of water area due to either built-up area or cloud shadow.

Having outstanding performance of water index with deep CNN models, motivates us to explore the method with other recent deep CNNs like DenseNet. As shallow water mapping can be further improvised by exploring more about the band combination. As Sentinel-2 also provides another SWIR band at $2190 \mathrm{~nm}$ and Cirrus SWIR band, it would be interesting to explore their impact on water index and cirrus clouds separation respectively. It would also be interesting to forgo the pre-calculation of water indices and instead supply all data to the deep neural network - i.e., all 12 channels - and allow the network to directly learn appropriate index like 
functions from the full data set. Similarly we expect that providing a learning function with information on the geographical area is likely to boost predictive performance.

\section{CONCLUSION}

In this paper we proposed an automatic flood detection technique by fusing a modified water index technique and deep CNN model to train and test against labelled sentinel-2 high resolution images. The modified water index is the combination of two indices, green/SWIR and blue/NIR in order to reduce the impact of clouds and cloud shadow for shallow water detection. The result showed that in compare to 5 other indices that is NDWI, MNDWI, AWEI, Mishra \& Prasad's [20] and Li et al.'s [17], our index performed the best, when trained with the VGG16 model. The outstanding results also shows that automatic flood detection is possible when an appropriate water index technique is being used. In our later work, we would also like to extend our approach with flood water segmentation, in order to have better flood mapping along with flood detection.

\section{REFERENCES}

[1] Benjamin Bischke, Patrick Helber, Erkan Basar, Simon Brugman, Zhengyu Zhao and Konstantin Pogorelov. 2019. The Multimedia Satellite Task at MediaEval 2019: Flood Severity Estimation. In Proc. of the MediaEval 2019 Workshop. SophiaAntipolis, France.

[2] Jia Deng, Wei Dong, Richard Socher, Li-Jia Li, Kai Li, and Li Fei-Fei. 2009. Imagenet: A large-scale hierarchical image database. In 2009 IEEE conference on computer vision and pattern recognition. Ieee, 248-255.

[3] Yun Du, Yihang Zhang, Feng Ling, Qunming Wang, Wenbo Li, and Xiaodong Li. 2016. Water bodies' mapping from Sentinel-2 imagery with modified normalized difference water index at $10-\mathrm{m}$ spatial resolution produced by sharpening the SWIR band. Remote Sensing 8, 4 (2016), 354.

[4] Arabi Mohammed El Amin, Qingjie Liu, and Yunhong Wang. 2016. Convolutional neural network features based change detection in satellite images. In First International Workshop on Pattern Recognition, Vol. 10011. International Society for Optics and Photonics, 100110W.

[5] Gudina L Feyisa, Henrik Meilby, Rasmus Fensholt, and Simon R Proud. 2014. Automated Water Extraction Index: A new technique for surface water mapping using Landsat imagery. Remote Sensing of Environment 140 (2014), 23-35.

[6] Gang Fu, Changjun Liu, Rong Zhou, Tao Sun, and Qijian Zhang. 2017. Classification for high resolution remote sensing imagery using a fully convolutional network. Remote Sensing 9, 5 (2017), 498.

[7] Geoffrey E Hinton and Ruslan R Salakhutdinov. 2006. Reducing the dimensionality of data with neural networks. science 313, 5786 (2006), 504-507.

[8] Renaud Hostache, Patrick Matgen, Guy Schumann, Christian Puech, Lucien Hoffmann, and Laurent Pfister. 2009. Water level estimation and reduction of hydraulic model calibration uncertainties using satellite SAR images of floods. IEEE Transactions on Geoscience and Remote Sensing 47, 2 (2009), 431-441.

[9] Fan Hu, Gui-Song Xia, Jingwen Hu, and Liangpei Zhang. 2015. Transferring deep convolutional neural networks for the scene classification of high-resolution remote sensing imagery. Remote Sensing 7, 11 (2015), 14680-14707.

[10] Yangqing Jia, Evan Shelhamer, Jeff Donahue, Sergey Karayev, Jonathan Long, Ross Girshick, Sergio Guadarrama, and Trevor Darrell. 2014. Caffe: Convolutional architecture for fast feature embedding. In Proceedings of the 22nd ACM international conference on Multimedia. ACM, 675-678.

[11] Sebastiaan N Jonkman and Ilan Kelman. 2005. An analysis of the causes and circumstances of flood disaster deaths. Disasters 29, 1 (2005), 75-97.

[12] Sascha Klemenjak, Björn Waske, Silvia Valero, and Jocelyn Chanussot. 2012. Unsupervised river detection in RapidEye data. In 2012 IEEE International Geoscience and Remote Sensing Symposium. IEEE, 6860-6863.

[13] Alex Krizhevsky, Ilya Sutskever, and Geoffrey E Hinton. 2012. Imagenet classification with deep convolutional neural networks. In Advances in neural information processing systems. 1097-1105.

[14] Yann LeCun, Yoshua Bengio, et al. 1995. Convolutional networks for images, speech, and time series. The handbook of brain theory and neural networks 3361, 10 (1995), 1995.

[15] Yann LeCun, Yoshua Bengio, and Geoffrey Hinton. 2015. Deep learning. nature 521, 7553 (2015), 436

[16] Erzhu Li, Junshi Xia, Peijun Du, Cong Lin, and Alim Samat. 2017. Integrating multilayer features of convolutional neural networks for remote sensing scene classification. IEEE Transactions on Geoscience and Remote Sensing 55, 10 (2017), 5653-5665.

[17] Na Li, Arnaud Martin, and Rémi Estival. 2017. An automatic water detection approach based on Dempster-Shafer theory for multi-spectral images. In 2017 20th International Conference on Information Fusion (Fusion). IEEE, 1-8.

[18] Stuart K McFeeters. 1996. The use of the Normalized Difference Water Index (NDWI) in the delineation of open water features. International journal of remote sensing 17, 7 (1996), 1425-1432.

[19] Renato Miceli, Igor Sotgiu, and Michele Settanni. 2008. Disaster preparedness and perception of flood risk: A study in an alpine valley in Italy. Fournal of environmental psychology 28, 2 (2008), 164-173.

[20] Kshitij Mishra and P Prasad. 2015. Automatic extraction of water bodies from Landsat imagery using perceptron model. Journal of Computational Environmental Sciences 2015 (2015).

[21] Keiller Nogueira, Waner O Miranda, and Jefersson A Dos Santos. 2015. Improving spatial feature representation from aerial scenes by using convolutional networks. In 2015 28th SIBGRAPI Conference on Graphics, Patterns and Images. IEEE, 289296.

[22] Eduardo R Oliveira, Leonardo Disperati, Luca Cenci, Luísa Gomes Pereira, and Fátima L Alves. 2019. Multi-Index Image Differencing Method (MINDED) for Flood Extent Estimations. Remote Sensing 11, 11 (2019), 1305

[23] Maxime Oquab, Leon Bottou, Ivan Laptev, and Josef Sivic. 2014. Learning and transferring mid-level image representations using convolutional neural networks. In Proceedings of the IEEE conference on computer vision and pattern recognition. 1717-1724.

[24] Karen Simonyan and Andrew Zisserman. 2014. Very deep convolutional networks for large-scale image recognition. arXiv preprint arXiv:1409.1556 (2014).

[25] Krishna Kant Singh and Akansha Singh. 2017. Identification of flooded area from satellite images using Hybrid Kohonen Fuzzy C-Means sigma classifier. The Egyptian fournal of Remote Sensing and Space Science 20, 1 (2017), 147-155.

[26] Laurence C Smith. 1997. Satellite remote sensing of river inundation area, stage, and discharge: A review. Hydrological processes 11, 10 (1997), 1427-1439.

[27] Guerschman JP Ticehurst C and Chen Y. 2014. The Strengths and Limitations in Using the Daily MODIS Open Water Likelihood Algorithm for Identifying Flood Events. Remote Sensing 6, 12 (2014), 11791-11809.

[28] Yunchao Wei, Wei Xia, Min Lin, Junshi Huang, Bingbing Ni, Jian Dong, Yao Zhao, and Shuicheng Yan. 2015. HCP: A flexible CNN framework for multi-label image classification. IEEE transactions on pattern analysis and machine intelligence 38, 9 (2015), 1901-1907.

[29] Hanqiu Xu. 2006. Modification of normalised difference water index (NDWI) to enhance open water features in remotely sensed imagery. International journal of remote sensing 27, 14 (2006), 3025-3033. 